\title{
The Effect of Tempol Administration on the Aortic Contractile Responses in Rat Preeclampsia Model
}

\author{
Mohammad Sharif Talebianpoor ${ }^{1,2}$ and Hossein Mirkhani ${ }^{1,3}$ \\ ${ }^{1}$ Department of Pharmacology, Shiraz University of Medical Sciences, Shiraz 71348-53185, Iran \\ ${ }^{2}$ Herbal Medicine Research Center, School of Medicine, Yasouj University of Medical Sciences, Yasouj, Iran \\ ${ }^{3}$ Medicinal and Natural Products Chemistry Research Center, Shiraz University of Medical Sciences, Shiraz, Iran \\ Correspondence should be addressed to Hossein Mirkhani, mirkhanh@sums.ac.ir \\ Received 7 April 2012; Accepted 17 July 2012 \\ Academic Editors: G. Biala, M. Brunner, and K. Cimanga
}

Copyright (C) 2012 M. S. Talebianpoor and H. Mirkhani. This is an open access article distributed under the Creative Commons Attribution License, which permits unrestricted use, distribution, and reproduction in any medium, provided the original work is properly cited.

It is reported that reactive oxygen species production has a critical role in the manifestations and complications of preeclampsia. In the present study, the effect of tempol on the response changes of aortic rings of preeclamptic rats has been studied. Preeclamptic rats (induced by L-NAME) were treated with three different oral doses of tempol $(20,60$ and $180 \mathrm{mg} / \mathrm{kg} / \mathrm{day})$ from the Day 10 of gestation. Systolic blood pressure, plasma malondialdehyde and 8-isoprostane and the vascular effects of phenylephrine, calcium, acetylcholine and diazoxide were the studied parameters. L-NAME administration resulted in hypertension, proteinuria, increased oxidative stress markers, increased vascular sensitivity to phenylephrine and decreased sensitivity to acetylcholine in pregnant rats. No significant changes in response to calcium and diazoxide were observed. Tempol at doses of 20 and $60 \mathrm{mg} / \mathrm{kg} / \mathrm{day}$ significantly reversed these changes but at a high dose $(180 \mathrm{mg} / \mathrm{kg} /$ day $)$, it had no significant effect and in some cases intensified the effect. These results revealed that in the experimental preeclampsia, the sensitivity of rat aorta to alpha- adrenergic receptor agonists was increased and its endothelium-dependent relaxation was decreased. Tempol at lower used doses reduced the blood pressure and oxidative stress and restored the normal responsiveness of vascular tissue in preeclamptic rats.

\section{Introduction}

Preeclampsia (PE) is defined as the onset of hypertension and proteinuria after 20 weeks of gestation in previously normotensive nonproteinuric pregnant women. This syndrome is a leading cause of maternal and fetal morbidity and mortality [1]. The etiology of this disorder is not known. Poor placental perfusion along with the endothelial cell dysfunction and a disturbed balance of angiogenic factors may all contribute to this disorder. Poor placental perfusion is a stimulus of reactive oxygen species (ROS) production. It is believed that the latter has a critical role in the manifestations and complications of PE [2].

In contrast to normal pregnancy, preeclampsia is characterized by generalized vasoconstriction, increased systemic vascular resistance, increased pressor response to vasoconstrictor agonists such as angiotensin II, endothelin, thromboxane, and widespread vascular endothelial damage [3].
To study the various aspects of PE, several animal models have been proposed [4]. One of the most popular models is administration of $N(\mathrm{G})$-nitro-L-arginine methyl ester (L-NAME) as a nitric oxide synthase (NOS) inhibitor during mid to late period of gestation of the animal (usually rat). NOS inhibition results in pathological changes similar to those observed in women with preeclampsia, such as hypertension, proteinuria, severe renal vasoconstriction, thrombocytopenia, and intrauterine growth retardation [5].

The present study was conducted to identify some mechanisms of vascular response changes in experimental $\mathrm{PE}$ model. Moreover, to investigate the role of oxidative stress in these changes, the effect of tempol, a synthetic antioxidant and a superoxide dismutase mimetic agent, was also studied. The used agents to clarify these alterations were phenylephrine (an alpha-adrenergic agonist), calcium (as an spasmogen which enters the cell via voltageoperated calcium channels), acetylcholine (endothelial NO 
releaser) and diazoxide (an ATP-dependent potassium channel opener).

\section{Methods}

2.1. Animals. Female Sprague-Dawley rats weighing 250$300 \mathrm{~g}$ were used. The animals were kept at controlled temperature $\left(25^{\circ} \mathrm{C}\right)$ and $12 \mathrm{~h}$ light- $12 \mathrm{~h}$ dark condition. They had free access to food and tap water (except after Day 10 of pregnancy in which the drinking water was modified as stated below). The female rats were mated at night with male Sprague-Dawley rats. Day 0 of pregnancy was defined as the day when the vaginal plaque was seen. On Day 10 of pregnancy, the rats were divided into five groups: group I consisted of normal pregnant animals that received only tap water. Rats in group II were treated with L-NAME $50 \mathrm{mg} / \mathrm{kg} /$ day dissolved in drinking water; rats in groups III, $\mathrm{IV}$, and $\mathrm{V}$ were treated with L-NAME $(50 \mathrm{mg} / \mathrm{kg} / \mathrm{day})$ plus tempol 20,60, and $180 \mathrm{mg} / \mathrm{kg} /$ day, respectively. Both agents were administered through drinking water.

All the animal procedures were approved by the Ethics Committee of Shiraz University of Medical Sciences.

2.2. Drugs and Chemicals. L-NAME was purchased from Alexis Biochemicals (USA). Tempol, phenylephrine, acetylcholine, diazoxide, 1,1,3,3-tetraethoxypropan (TEP), trichloroacetic acid (TCA), and thiobarbituric acid (TBA) were purchased from Sigma Chemical Company. 8-isoprostane ELISA kit was purchased from Cayman Chemical Company (Ann Arbor, MI, USA). All the chemicals used for preparation of physiological salt solution (PSS) were of analytical grade.

L-NAME and tempol were dissolved in animals' drinking water. Phenylephrine and acetylcholine were dissolved in distilled water to make $10^{-3} \mathrm{M}$ stock solution. Diazoxide was dissolved in dimethyl sulfoxide (DMSO). Further dilutions were made by adding distilled water.

\subsection{Measurement of Blood Pressure, Urine Protein, Plasma} Malondialdehyde, and 8-Isoprostane. Systolic blood pressure was measured in all groups on gestational Days 10, 13, 15, 18, and 21 by an automated sphygmomanometer with a tail-cuff device (ML 125/R, ADInstruments, Australia). Measuring was made on pretrained rats placed in a plexiglass restrainer. Ten measurements with $30 \mathrm{~s}$ intervals were done. The first three were ignored and the mean of the last seven reads was calculated.

To ensure about PE establishment, urine protein of group 1 (normal pregnant rats) and group 2 (pregnant rats received L-NAME) was assayed on Day 20 of gestation. To accomplish this, animals were placed in metabolic cages, $24 \mathrm{~h}$ urine was collected, and its protein content was measured based on Bradford method [6].

On gestational Day 20, the rats were sedated with ether, and a blood sample was taken via tail artery. Then it was centrifuged at $1000 \mathrm{~g}$ for $10 \mathrm{~min}$ and its plasma was collected and immediately frozen to $-80^{\circ} \mathrm{C}$ until the day of analysis.
Plasma malondialdehyde (MDA), a lipid peroxidation end product, was assessed based on the reaction of thiobarbituric acid with MDA. Malondialdehyde (MDA) concentration was determined by comparison to a standard curve of TEP. Standard curve was made using serial dilution of TEP to yield the following test concentrations: $0,1,2,2.5,5$, and $10 \mu \mathrm{M} .0 .5 \mathrm{~mL}$ of plasma or standard solutions was taken in a test tube and $2 \mathrm{~mL}$ of the TBA-TCA (TBA-TCA reagent: $0.375 \% \mathrm{w} / \mathrm{v}$ TBA, $15 \% \mathrm{w} / \mathrm{v}$ TCA, and $0.25 \mathrm{~N} \mathrm{HCl}$ ) solution were added. The mixture was heated in a water bath $(90$ $100^{\circ} \mathrm{C}$ ) for $15 \mathrm{~min}$, cooled in a cold water bath for $10 \mathrm{~min}$, and then centrifuged at $2000 \mathrm{~g}$ for $15 \mathrm{~min}$. The absorbance of solution was read spectrophotometrically at $535 \mathrm{~nm}$ [7].

Plasma 8-isoprostane concentration was measured by an enzyme immunoassay kit according to the manufacturer's instructions.

2.4. Isolated Aorta Studies. On Day 22 of gestation, the animals were sacrificed under ether anesthesia. The thorax was opened. The thoracic aorta was removed carefully, placed in a Petri dish containing PSS and divided into four pieces of about 2-3 mm length. The endothelium of three aortic pieces was removed by rolling a fine needle (gauge 18) inside the vessel. The endothelium of one piece was left intact to investigate endothelium-dependent relaxation by acetylcholine. Each aortic ring was mounted between two parallel wires in a four-chamber organ bath at $37^{\circ} \mathrm{C}$. The resting tension was adjusted to $0.5 \mathrm{~g}$. The PSS contained (in $\mathrm{mM}) \mathrm{NaCl} 118, \mathrm{KCl} 4.8, \mathrm{KH}_{2} \mathrm{PO}_{4} 1.2, \mathrm{CaCl}_{2} 2.5, \mathrm{MgSO}_{4}$ $1.2, \mathrm{NaHCO}_{3} 25$, and $\mathrm{D}$-glucose 11 . Its $\mathrm{pH}$ was adjusted to 7.4. The solution was bubbled with $95 \% \mathrm{O}_{2} / 5 \% \mathrm{CO}_{2}$ and changed every $10 \mathrm{~min}$. The tension of the rings was recorded via a force transducer (K 30, Hugo Sachs Elektronik, Germany) and an amplifier (Transducer-Amplifier Module Type 705/1, Hugo Sachs Elektronik, Germany) on a PC software (HSE-ACAD, Hugo Sachs Electronik, Germany). The aortic rings were allowed to equilibrate for $1 \mathrm{hr}$.

To study the changes in sensitivity to alpha-adrenergic receptor agonists, the aortic ring was exposed to cumulative concentrations of phenylephrine. The $\mathrm{pEC}_{50}$ (negative logarithm of effective concentration which produced 50 percent of maximum response) and maximum response were calculated.

To investigate the state of voltage-dependent calcium channels, the tissue was incubated in high potassium $(100 \mathrm{mM})$ calcium-free PSS for 60 minutes. The high- $\mathrm{KCl}$ depolarizing solution was prepared by equimolar substitution of $\mathrm{NaCl}$ with $\mathrm{KCl}$. Thereafter, increasing concentrations of calcium were added to the organ bath and the $\mathrm{pEC}_{50}$ and maximum response were calculated.

To study the endothelial cells capability to release nitric oxide (NO), another aortic ring was contracted with submaximal concentration (60-70\% of maximal response) of phenylephrine. After reaching the plateau, the tissue was treated with cumulative concentrations of acetylcholine. The $\mathrm{pIC}_{50}$ (negative logarithm of concentration at which the ring relaxed to $50 \%$ of its initial contraction) and maximum relaxation were calculated. 


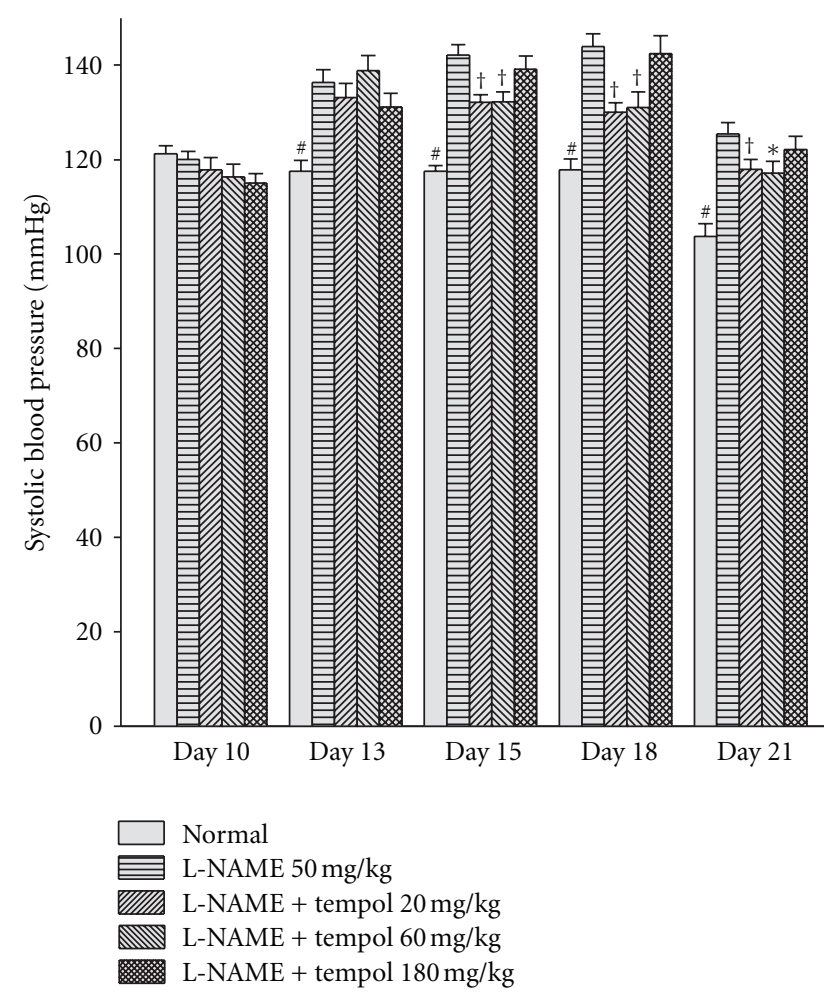

FIGURE 1: Blood pressure of rats on Days 10 (before administration of any agents), 13, 15, 18, and 21 of gestation ( $n=10-15)$. Each value represents mean \pm SEM. Statistical comparisons were made between obtained value of each group with that of preeclamptic (LNAME treated) group. ${ }^{*} P<0.05 ;{ }^{\dagger} P<0.01 ;{ }^{\#} P<0.001$.

To study the responsiveness of vascular ATP-dependent potassium channels, another aortic ring was exposed to $\mathrm{KCl}$ $(30 \mathrm{mM})$. When maximum tension was reached, cumulative concentrations of diazoxide were added to the organ bath and $\mathrm{IC}_{50}$ and maximum response were calculated.

2.5. Statistical Analysis. All the values were expressed as the mean \pm SEM (standard error of the mean). Maximum contractile responses to phenylephrine and calcium were expressed as developed tension (g). Relaxation responses to acetylcholine and diazoxide were expressed as a percentage of relaxation of the maximum contractions. $\mathrm{pEC}_{50}$ and $\mathrm{pIC}_{50}$ values were calculated based on the best-fit method using Curve Expert software (Ver. 1.3). The data were analyzed using unpaired Student's $t$-test and analysis of variance (ANOVA) followed by a posthoc Dunnett test. Differences were considered to be statistically significant when $P$ value was $<0.05$.

\section{Results}

Before the administration of L-NAME, systolic blood pressure and 24-hour urine protein did not differ between normal and preeclamptic groups. The SBP was significantly higher $(P<0.001)$ in the L-NAME treated group compared to the control group on Days 13, 15, 18 and 21 of gestation
(Figure 1). The comparison also showed higher 24 hour urinary protein in the L-NAME treated group (5.29 \pm $0.28 \mathrm{mg}$ versus $3.66 \pm 0.27 \mathrm{mg}, P<0.001$ ).

Tempol at 20 and $60 \mathrm{mg} / \mathrm{kg}$ doses significantly lowered SBP on Days 15, 18, and 21 in the preeclamptic rats. In contrast, the highest dose of tempol, that is, $180 \mathrm{mg} / \mathrm{kg}$, had no significant effect on this parameter (Figure 1).

Plasma level of 8-isoprostane and MDA as indices of whole body oxidative stress was higher in the preeclamptic rats $(P<0.001)$. Tempol treatment at doses 20 and 60 but not $180 \mathrm{mg} / \mathrm{kg} /$ day reduced the plasma concentration of MDA and 8-isoprostane (Figure 2).

The aortic rings of preeclamptic rats were more sensitive to the contractile effect of phenylephrine (Table 1). However, there was no significant difference in phenylephrineinduced maximal tension between preeclamptic and normal rats (Table 1). Aortic rings of tempol-treated preeclamptic rats (all 3 doses) showed significantly reduced sensitivity to phenylephrine (Figure 3(a), Table 1). The maximum phenylephrine-induced contraction was significantly lower in the preeclamptic animals treated by 20 and $60 \mathrm{mg} / \mathrm{kg}$ of tempol (Table 1).

The contractile response of the aorta to calcium did not differ among groups (Figure 3(b), Table 1). However, calcium-induced maximum tension (and not its $\mathrm{pEC}_{50}$ ) in preeclamptic animals fed with tempol was remarkably higher than that of the preeclamptic controls, but statistically significant difference was only reached by the dose of $180 \mathrm{mg} / \mathrm{kg}$ (Table 1).

The potency and maximal effect of acetylcholine to relax aortic rings precontracted by phenylephrine were lower in preeclamptic rats (Figure 4(a), Table 1). Aortic rings of preeclamptic animals treated by tempol 20 and 60 but not $180 \mathrm{mg} / \mathrm{kg}$ showed significantly higher sensitivity to acetylcholine compared to the preeclamptic controls. Also, the maximal acetylcholine-induced relaxation was significantly greater in animals receiving these doses (Figure 4(a), Table 1).

$\mathrm{pIC}_{50}$ and maximum relaxing effect of diazoxide on KClinduced aortic contraction did not differ among the studied groups (Figure 4(b), Table 1).

\section{Discussion}

In the present study, the action of tempol as a synthetic antioxidant on blood pressure and responsiveness to some vasodilators and vasoconstrictors is evaluated. Tempol can penetrate cell membranes and reacts with both intracellular and extracellular oxygen-free radicals [8]. These properties make tempol attractive for treatment of cardiovascular diseases associated with oxidative stress [9].

Administration of L-NAME from Day 10 of gestation induced two hallmarks of PE, that is, hypertension (Figure 1) and proteinuria. Also, plasma MDA and 8-isoprostane as markers of oxidative stress rose significantly in the LNAME-treated pregnant rats (Figure 2). In pregnant rats, it was shown that treatment with L-NAME decreased plasma $\mathrm{NO}$, prostacycline, and angiotensin II and increased plasma endothelin and thromboxane A2 [10]. It was also reported 

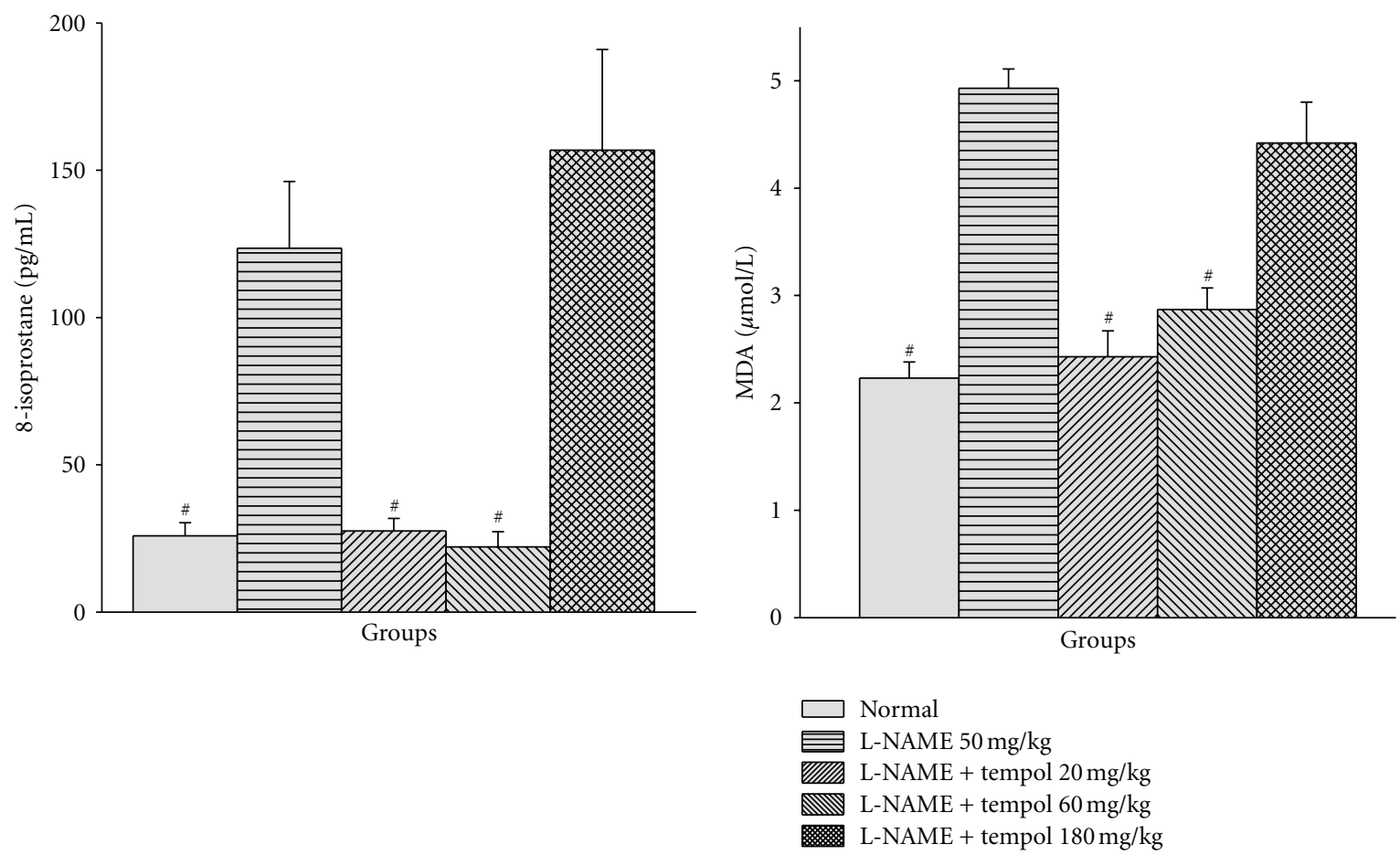

(a)

(b)

Figure 2: Concentrations of the plasma 8-isoprostane (a) and MDA (b) on Day 20 of gestation $(n=10-12)$. Each value represents mean \pm SEM. Statistical comparisons were made between obtained value of each group with that of preeclamptic (L-NAME treated) group. ${ }^{\#} P<$ 0.001 .

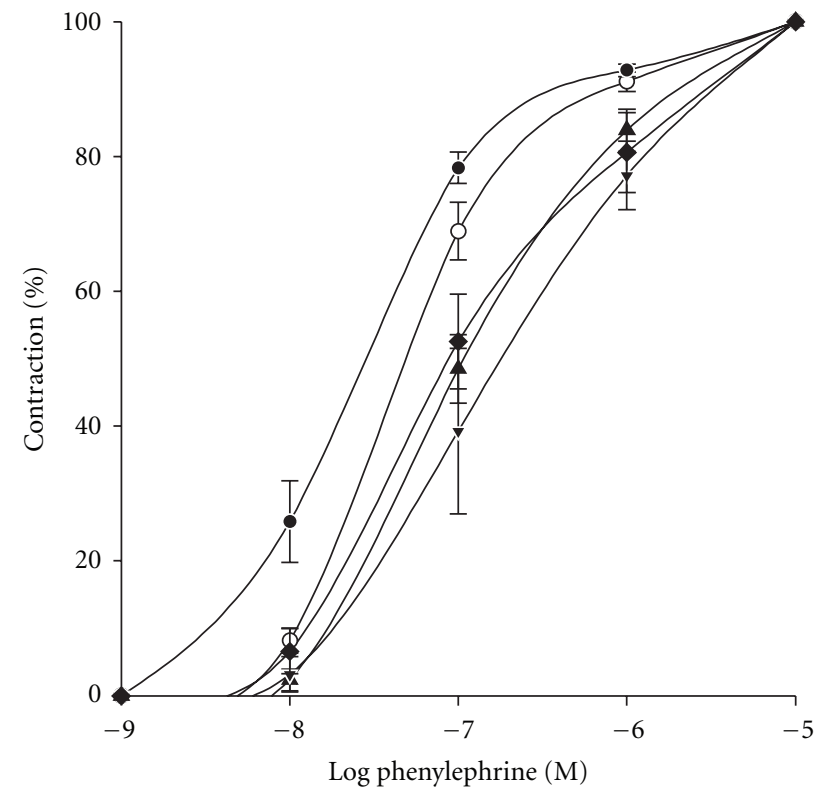

(a)

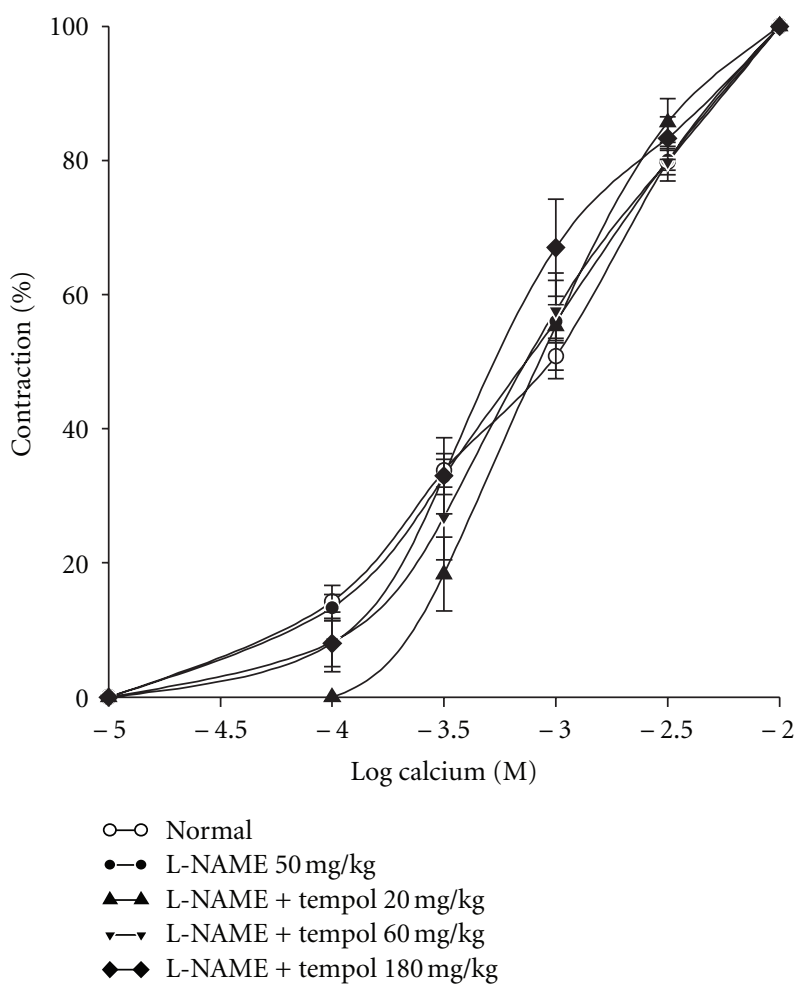

(b)

FIGURE 3: Contractile responses of endothelium-denuded aortic rings of normal and preeclamptic (L-NAME treated) rats to phenylephrine (a) and calcium (b). Symbols represent means \pm SEM of 8-10 experiments. For statistical analysis, see Table 1. 


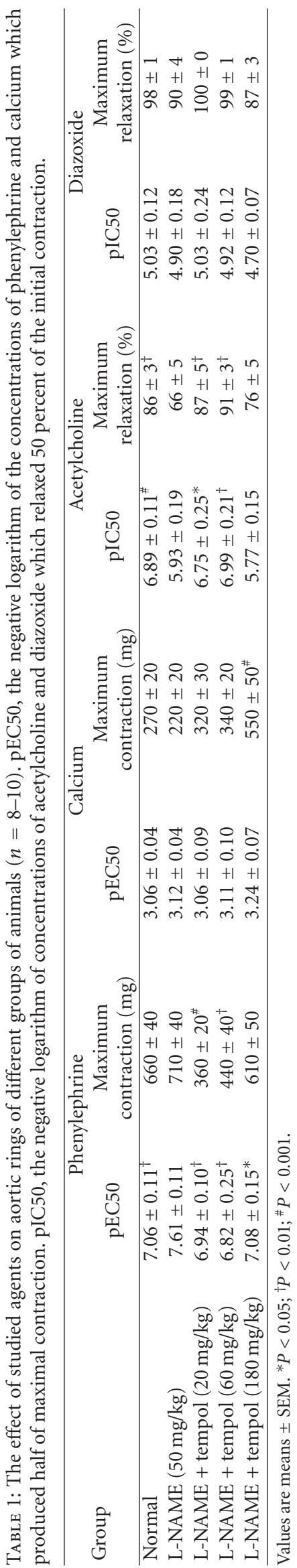




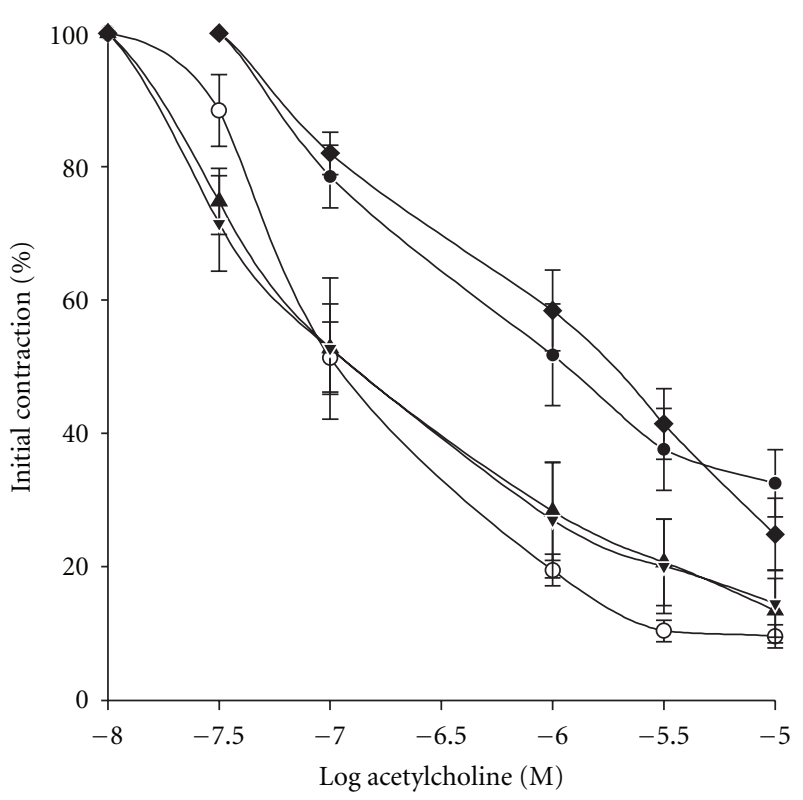

(a)

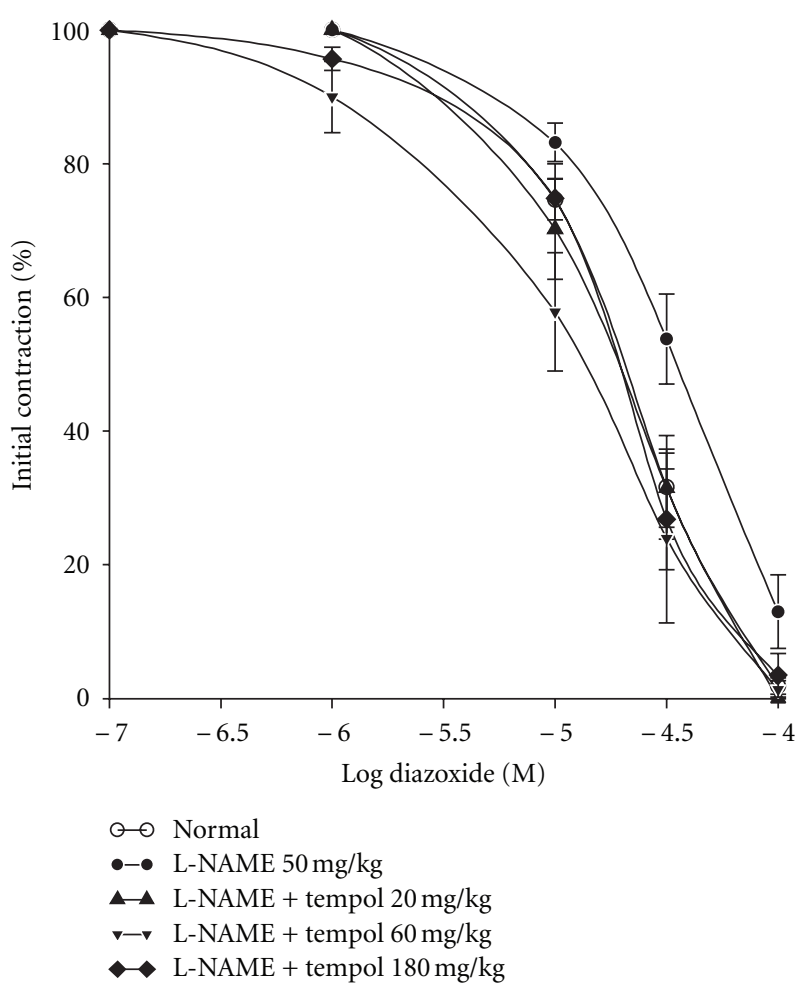

(b)

FIGURE 4: Relaxation responses of aortic rings of normal and preeclamptic rats (L-NAME treated). (a) Vasorelaxant effect of acetylcholine on phenylephrine-induced contraction; (b) vasorelaxant effect of diazoxide on potassium (30 mM)-induced contraction. Symbols represent means \pm SEM of 8-10 experiments. For statistical analysis, see Table 1.

that hypertension induced by chronic NO deficiency is associated with increased oxidative stress $[11,12]$.

Tempol at doses of 20 and $60 \mathrm{mg} / \mathrm{kg} /$ day significantly reduced $\mathrm{BP}$ and plasma MDA and 8-isoprostane (Figures 1 and 2). Tempol is a synthetic superoxide dismutase (SOD) mimetic agent. SOD catalyzes the conversion of $\mathrm{O}_{2}{ }^{-}$to $\mathrm{H}_{2} \mathrm{O}_{2}$, which can be turned into water by catalase or the glutathione peroxidase system. Although SOD is the firstline physiological defense against oxidative stress, superoxide anions reaction with NO to form peroxynitrite, is threefold faster than their reaction with SOD which forms hydrogen peroxide [8]. Tempol potentiates antioxidant defense and may increase NO bioavailability. It has been reported that chronic tempol administration to hypertensive rats is accompanied by increased endothelium-dependent vasodilation [13-15]. Nevertheless, since in the present study the preeclamptic animals had been treated by L-NAME, a wellknown NO synthesis inhibitor, rising NO level by tempol is questionable. So, the antihypertensive effect of tempol may be produced by NO-independent pathways. The effect of tempol on lowering hypertension and proteinuria in a genetic mouse model of preeclampsia has also been reported by Hoffmann et al. [16].

High dose tempol $(180 \mathrm{mg} / \mathrm{kg} /$ day $)$ decreased neither blood pressure nor markers of oxidative stress. A similar result has been reported by Preti et al. [17]. As stated above, tempol changes the highly reactive $\mathrm{O}_{2}{ }^{-}$to $\mathrm{H}_{2} \mathrm{O}_{2}$. Although,
$\mathrm{H}_{2} \mathrm{O}_{2}$ can contribute to the vasodilatory effects of tempol, $\mathrm{H}_{2} \mathrm{O}_{2}$ excess may induce vascular contraction $[18,19]$. It is reported that a high concentration of $\mathrm{H}_{2} \mathrm{O}_{2}$ may inactivate NO [20].

Aortic rings of $\mathrm{PE}$ animals were more sensitive to the contractile effect of phenylephrine. However, maximuminduced contraction by this drug did not change in $\mathrm{PE}$ (Figure 3(a), Table 1). So, it seems that alpha-adrenergic receptors interaction with phenylephrine is changed in the experimental PE but the peak intracellular calcium increment or the interaction of contractile myofilaments is not. So, this change may be involved in the observed hypertension in PE animals. Aortic rings of tempol-treated $\mathrm{PE}$ animals show decreased sensitivity to phenylephrine. This effect can be seen even by tempol $180 \mathrm{mg} / \mathrm{kg}$ dose, a contradictory result with the observed effects of this dose on blood pressure and oxidative stress. We have no explanation for this discrepancy.

Entrance of calcium via calcium voltage operated channels and its effect on the contractile state did not change in L-NAME-induced PE (Figure 3(b), Table 1). So, it can be concluded that these channels have no role in PE hypertension. Also, the sensitivity of vascular contraction apparatus to calcium did not change in PE. Tempol administration to PE animals did not change the sensitivity of the vascular tissues to calcium. However, tempol at its highest dose significantly increased the maximum calcium-induced contraction. 
The potency and maximum effect of acetylcholine to relax aortic rings were diminished significantly in PE rats (Figure 4(a), Table 1). This effect can be attributed to the oxidative stress in the endothelial cells which results in endothelial dysfunction. In our experiments, the animals had no access to L-NAME since the morning of the day of tissue studies. Moreover, after animal sacrifice, the aortic rings were held in PSS for about 2-3 hr. So, the studied vascular rings were free from the inhibitory effect of L-NAME on NO synthesis, and the above mentioned observation was due to the endothelial dysfunction produced by L-NAME administration during pregnancy. In accordance with the findings on blood pressure and oxidative stress, tempol treatment (20 and $60 \mathrm{mg} / \mathrm{kg}$ ) improved the responses to acetylcholine in the preeclamptic rat aortas, denoting improvements in endothelial function in preeclamptic rats (Figure 4(a), Table 1). Decreased production and/or inactivation of superoxide anions by tempol can protect the endothelial cells, enhance the bioavailability of NO released from the endothelium, and mediate the beneficial effects of tempol on vascular reactivity in preeclamptic rats [21-25]. It has been reported that chronic tempol treatment of hypertensive rats is accompanied by increased endothelium-dependent vasodilation [13-15]. Tempol at high dose (180 mg/kg/day) did not improve the ACh relaxation in L-NAME treated rats. This observation is similar to the results obtained in blood pressure and oxidative stress and may have the similar basis.

The vasodilatory effect of diazoxide, an ATP-dependent potassium channel opener, on aortic rings of $\mathrm{PE}$ and normal pregnant rats was similar. So, in the experimental PE, vascular ATP-dependent potassium channels have no role in hemodynamic changes. It has been reported that endothelium-dependent hyperpolarizing factor (EDHF) action on the vascular tissue is diminished in PE [26]. It opens ATP-dependent potassium channels. Based on our results, it can be concluded that the decreased action of EDHF may be due to the reduction of its production, and ATP-potassium channel condition has no contribution to this reported observation.

In conclusion, in the experimental PE induced by LNAME, blood pressure, oxidative stress markers, and the sensitivity of vascular tissue to contractile effects of alphaadrenergic receptors are increased, and the endotheliumdependent relaxation by acetylcholine is decreased. Tempol at 20 and $60 \mathrm{mg} / \mathrm{kg}$ doses reverses the observed changes but in the dose of $180 \mathrm{mg} / \mathrm{kg}$ it has no effect.

\section{Conflict of Interests}

The authors have declared that no conflict of interests exists.

\section{Acknowledgments}

The authors would like to thank Dr. O. Firuzi, Ms. M. Mojahed and Ms. R. Gholipour for their technical assistance. This work was supported by Grant no. 87-4011 from Vice-chancellory of Research, Shiraz University of Medical Sciences. Some part of this paper has been published before in an Iranian journal (Armaghan Danesh 2009; 14:17-29) in Persian language.

\section{References}

[1] M. Noris, N. Perico, and G. Remuzzi, "Mechanisms of disease: pre-eclampsia," Nature Clinical Practice. Nephrology, vol. 1, no. 2, pp. 98-114, 2005.

[2] M. Sedeek, J. S. Gilbert, B. B. Lamarca et al., "Role of reactive oxygen species in hypertension produced by reduced uterine perfusion in pregnant rats," American Journal of Hypertension, vol. 21, no. 10, pp. 1152-1156, 2008.

[3] R. A. Khalil and J. P. Granger, "Vascular mechanisms of increased arterial pressure in preeclampsia: Lessons from animal models," American Journal of Physiology-Regulatory Integrative and Comparative Physiology, vol. 283, no. 1, pp. R29-R45, 2002.

[4] E. Podjarny, G. Losonczy, and C. Baylis, "Animal models of preeclampsia," Seminars in Nephrology, vol. 24, no. 6, pp. 596606, 2004.

[5] H. M. Tanir, T. Sener, M. Inal, F. Akyuz, K. Uzuner, and E. Sivri, "Effect of quercetine and glutathione on the level of superoxide dismutase, catalase, malonyldialdehyde, blood pressure and neonatal outcome in a rat model of pre-eclampsia induced by NG-nitro-L-arginine-methyl ester," European Journal of Obstetrics Gynecology and Reproductive Biology, vol. 118, no. 2, pp. 190-195, 2005.

[6] M. M. Bradford, "A rapid and sensitive method for the quantitation of microgram quantities of protein utilizing the principle of protein dye binding," Analytical Biochemistry, vol. 72, no. 1-2, pp. 248-254, 1976.

[7] D. V. Hoyland and A. J. Taylor, "A review of the methodology of the 2-thiobarbituric acid test," Food Chemistry, vol. 40, no. 3, pp. 271-291, 1991.

[8] J. S. Beckman and W. H. Koppenol, "Nitric oxide, superoxide, and peroxynitrite: the good, the bad, and the ugly," American Journal of Physiology-Cell Physiology, vol. 271, no. 5, pp. C1424-C1437, 1996.

[9] U. Simonsen, R. Rodriguez-Rodriguez, T. Dalsgaard, N. H. Buus, and E. Stankevicius, "Novel approaches to improving endothelium-dependent nitric oxide-mediated vasodilatation," Pharmacological Reports, vol. 61, no. 1, pp. 105-115, 2009.

[10] C. Ma, Y. Zhuang, and Y. Xu, "The effect of inhibition of nitric oxide synthesis on vasoregulatory factors in pregnant rats," Zhonghua Fu Chan Ke Za Zhi, vol. 34, no. 9, pp. 521-524, 1999.

[11] H. Tsukahara, M. Hiraoka, R. Kobata et al., "Increased oxidative stress in rats with chronic nitric oxide depletion: measurement of urinary 8-hydroxy-2' -deoxyguanosine excretion," Redox Report, vol. 5, no. 1, pp. 23-28, 2000.

[12] J. Duarte, R. Jiménez, F. O’Valle et al., "Protective effects of the flavonoid quercetin in chronic nitric oxide deficient rats," Journal of Hypertension, vol. 20, no. 9, pp. 1843-1854, 2002.

[13] Y. Ozawa, K. Hayashi, T. Kanda et al., "Impaired nitric oxideand endothelium-derived hyperpolarizing factor-dependent dilation of renal afferent arteriole in Dahl salt-sensitive rats," Nephrology, vol. 9, no. 5, pp. 272-277, 2004.

[14] X. Gao, S. Belmadani, A. Picchi et al., "Tumor necrosis factoralpha induces endothelial dysfunction in Lepr(db) mice," Circulation, vol. 115, no. 2, pp. 245-254, 2007.

[15] L. Xiang, J. Dearman, S. R. Abram, C. Carter, and R. L. Hester, "Insulin resistance and impaired functional vasodilation in obese Zucker rats," American Journal of Physiology-Heart 
and Circulatory Physiology, vol. 294, no. 4, pp. H1658-H1666, 2008.

[16] D. S. Hoffmann, C. J. Weydert, E. Lazartigues et al., "Chronic tempol prevents hypertension, proteinuria, and poor fetoplacental outcomes in BPH/5 mouse model of preeclampsia," Hypertension, vol. 51, no. 4, pp. 1058-1065, 2008.

[17] S. C. Preti, V. da Cunha, D. V. Vassallo, and I. Stefanon, "The superoxide dismutase mimetic, tempol, reduces the bioavailability of nitric oxide and does not alter L-NAMEinduced hypertension in rats," Basic and Clinical Pharmacology and Toxicology, vol. 97, no. 1, pp. 29-34, 2005.

[18] N. Ardanaz, W. H. Beierwaltes, and P. J. Pagano, "Distinct hydrogen peroxide-induced constriction in multiple mouse arteries: potential influence of vascular polarization," Pharmacological Reports, vol. 60, no. 1, pp. 61-67, 2008.

[19] A. B. García-Redondo, A. M. Briones, A. E. Beltrán, M. J. Alonso, U. Simonsen, and M. Salaices, "Hypertension increases contractile responses to hydrogen peroxide in resistance arteries through increased thromboxane $\mathrm{A} 2, \mathrm{Ca}^{2+}$, and superoxide anion levels," Journal of Pharmacology and Experimental Therapeutics, vol. 328, no. 1, pp. 19-27, 2009.

[20] Y. F. Chen, A. W. Cowley Jr., and A. P. Zou, "Increased $\mathrm{H}_{2} \mathrm{O}_{2}$ counteracts the vasodilator and natriuretic effects of superoxide dismutation by tempol in renal medulla," American Journal of Physiology-Regulatory Integrative and Comparative Physiology, vol. 285, no. 4, pp. R827-R833, 2003.

[21] C. van Breemen, P. Aaronson, and R. Loutzenhiser, "Sodiumcalcium interactions in mammalian smooth muscle," Pharmacological Reviews, vol. 30, no. 2, pp. 167-208, 1978.

[22] E. Suematsu, M. Hirata, T. Hashimoto, and H. Kuriyama, "Inositol 1,4,5-trisphosphate releases $\mathrm{Ca}^{2+}$ from intracellular store sites in skinned single cells of porcine coronary artery," Biochemical and Biophysical Research Communications, vol. 120, no. 2, pp. 481-485, 1984.

[23] R. A. Khalil and C. van Breemen, "Mechanisms of calcium mobilization and homeostasis in vascular smooth muscle and their relevance to hypertension," in Hypertension: Pathophysiology, Diagnosis Management, J. H. Laragh and B. M. Brenner, Eds., pp. 523-540, Raven Press, New York, NY, USA, 1995.

[24] Y. M. Park, M. Y. Park, Y. L. Suh, and J. B. Park, "NAD(P)H oxidase inhibitor prevents blood pressure elevation and cardiovascular hypertrophy in aldosterone-infused rats," Biochemical and Biophysical Research Communications, vol. 313, no. 3, pp. 812-817, 2004.

[25] A. S. O. Adeagbo, I. G. Joshua, C. Falkner, and P. J. Matheson, "Tempol, an antioxidant, restores endotheliumderived hyperpolarizing factor-mediated vasodilation during hypertension," European Journal of Pharmacology, vol. 481, no. 1, pp. 91-100, 2003.

[26] L. C. Kenny, P. N. Baker, D. A. Kendall, M. D. Randall, and W. R. Dunn, "Differential mechanisms of endotheliumdependent vasodilator responses in human myometrial small arteries in normal pregnancy and pre-eclampsia," Clinical Science, vol. 103, no. 1, pp. 67-73, 2002. 

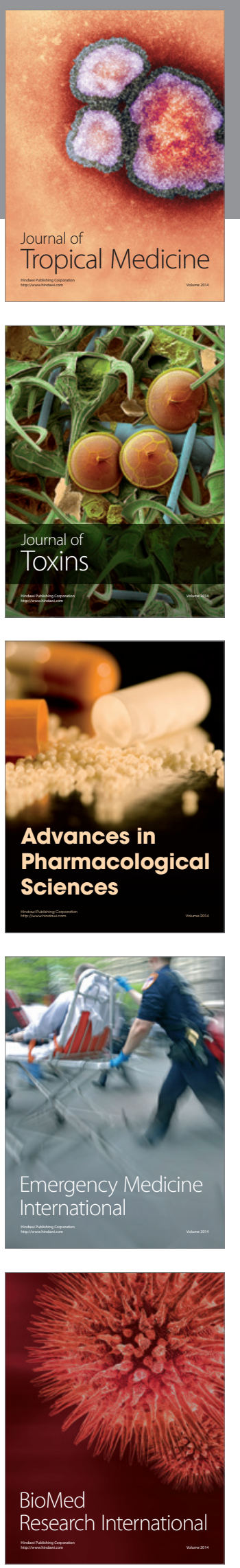
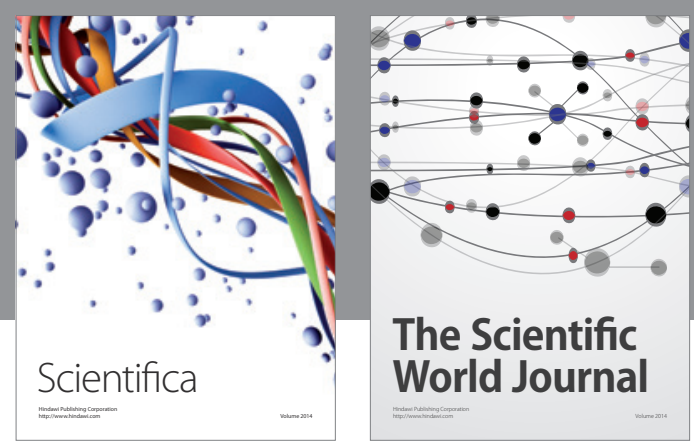

The Scientific World Journal
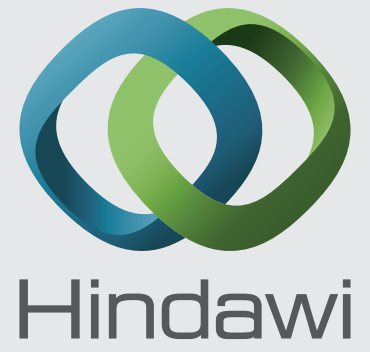

Submit your manuscripts at

http://www.hindawi.com
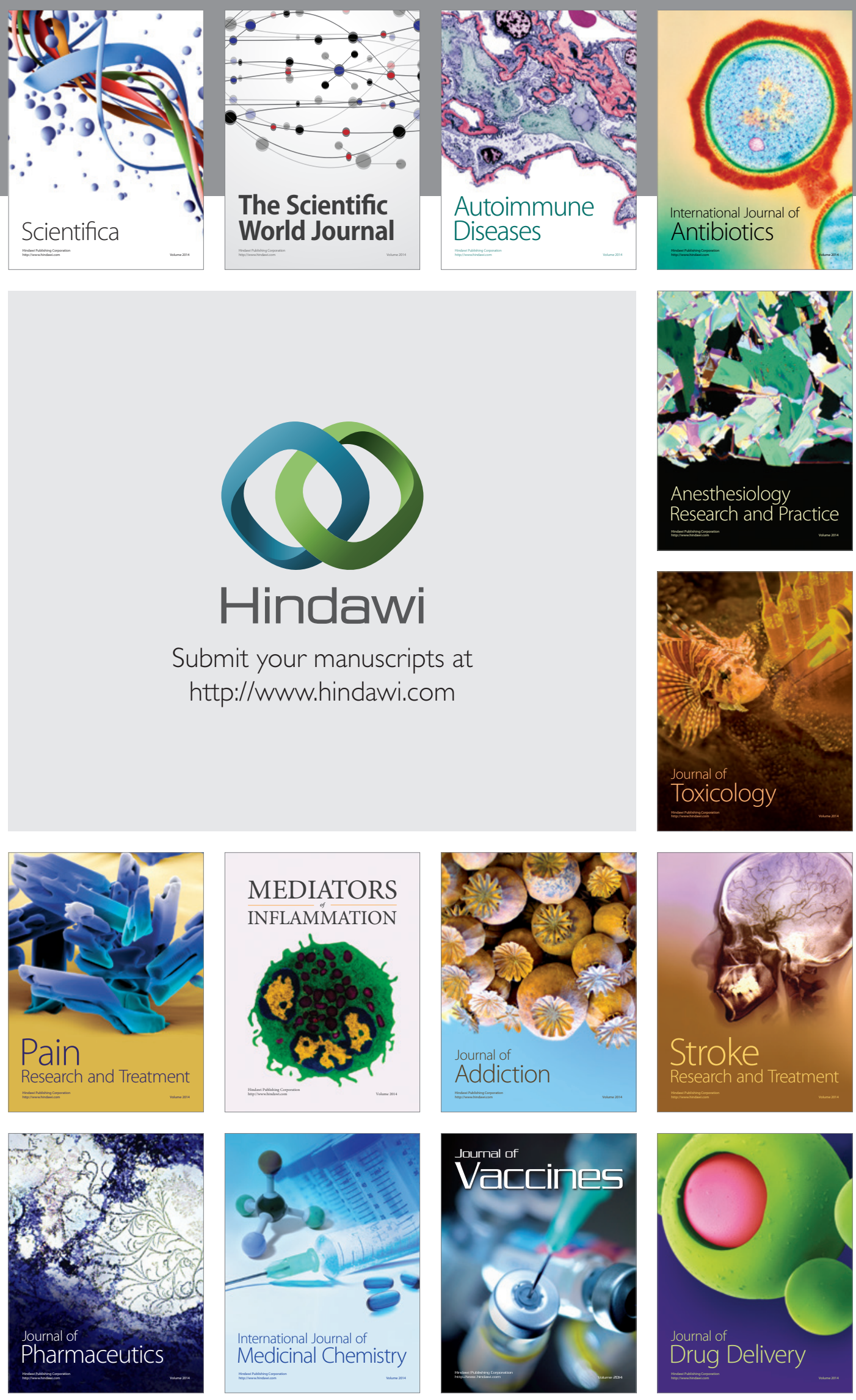